\title{
Effects of radiation and chemical reaction on MHD unsteady heat and mass transfer of nanofluid flow through a vertical plate
}

\author{
S.F. Ahmmed, R. Biswas* \\ Mathematics Discipline, Science, Engineering and Technology School, Khulna University, Khulna 9208, Bangladesh
}

Corresponding Author Email: rajibkumath11@gmail.com

https://doi.org/10.18280/mmc_b.870401

Received: 21 December 2017

Accepted: 10 March 2018

\section{Keywords:}

nanofluid, MHD, heat source, porous medium, chemical reaction

\begin{abstract}
In this paper we have reported the effects of radiation and chemical reaction on MHD unsteady heat and mass transfer of nanofluid flow through a vertical plate. The model equations are transformed into non-dimensional form by the as usual mathematical technique of transformation and the resultant non-dimensional partial differential equations are solved numerically by applying explicit finite difference technique. Then the numerical results have been calculated by computer programming languages Compaq Visual Fortran 6.6a which are significantly affected by the various dimensionless parameters such as Magnetic parameter, Schmidt number, Grashof number, Lewis number, Prandtl number, modified Grashof number, Dufour number, thermophoresis parameter, Brownian motion parameter, chemical reaction and radiation parameter on velocity, temperature and concentration profiles along with the skin friction coefficient, Nusselt number and Sherwood number. At the end, the obtained numerical results are plotted after stability test by using graphics software tecplot- 9 and discussed with the help of graphs.
\end{abstract}

\section{INTRODUCTION}

The concept of nanofluid was first established by Choi [1] in 1995 where the suspension of nanoparticles in a base fluid such as ethylene glycol, oil and water etc. are submitted. Nanofluid is the combination of simple fluid with nanoparticle of 1 to $100 \mathrm{~nm}$ size. These fluids are engineered colloidal suspensions of nanoparticles in a base fluid. Oxide ceramics $(\mathrm{CuO})$, carbide ceramics $(\mathrm{TiC}, \mathrm{SiC})$, metals $(\mathrm{Cu}, \mathrm{Ag}, \mathrm{Au})$, semiconductors $(\mathrm{SiC})$, carbon nanotubes and composite materials such as alloyed nanoparticles, $A l_{70} C u_{30}$. Water, ethylene glycol $(E G)$, engine oil $(E O)$, pump oil has been used as host liquids of nanofluids. Uddin et al. [2] have elaborated the evolution, applications and new theory of nanofluids. Unsteady three-dimensional MHD flow of a nano EyringPowell fluid past a convectively heated stretching sheet in the presence of thermal radiation, viscous dissipation and Joule heating has been explored by Mahanthesh et al. [3]. Also, Khan et al. [4] have elucidated the optimal solution of nonlinear heat and mass transfer in a two-layer flow with nano-Eyring-Powell fluid.

Mass transfer problems are of importance in many processes and have therefore received a considerable amount of attention. Mass can be transferred by diffusion of one component of a fluid mixture from a region of high concentration to one of low concentration. This is analogous to the transfer of heat from a high-temperature region to a low temperature region. In many mass transfer processes, heat transfer considerations arise owing to chemical reaction and are often due to the nature of the process. In processes such as drying, evaporation at the surface water body, energy transfer in a wet cooling tower and the flow in a desert cooler, heat and mass transfer occur simultaneously. MHD free convection and mass transfer flow through a vertical oscillatory porous plate with Hall, ion-slip currents and heat source in a rotating system has been capitalized by Hossain et al. [5]. Das et al. [6] have elaborated the Hall effects on an unsteady magneto-convection and radiative heat transfer past a porous plate. They have solved their governing equations by using fourth order RungeKutta- Fehlberg method with shooting technique. Effects of hall current, radiation and rotation on natural convection heat and mass transfer flow past a moving vertical plate has been illustrated by Seth et al. [7]. Effects of radiation and chemical reaction on MHD unsteady heat and mass transfer of Casson fluid flow past a vertical plate has been discussed by Biswas et al. [8]. Micropolar fluid behaviours on unsteady MHD heat and Mass transfer flow with constant heat and mass fluxes has been focused by Haque et al. [9]. Also, Haque et al. [10] have introduced the transient heat and mass transfer by mixed convection flow from a vertical porous plate with induced magnetic field, constant heat and mass fluxes.

Boundary layer phenomena occur when the influence of a physical quantity is restricted to small regions or near the confining boundaries. This phenomenon occurs when the nondimensional diffusion parameters the Reynolds number and the magnetic Reynolds number are large. The boundary layers are then the velocity and thermal or magnetic boundary layers and each thickness is inversely proportional to the square root of the associated diffusion number. When such contaminant is present in the fluid under consideration there takes place some chemical reaction e.g. air and benzene react chemically, such as water and sulphuric acid. During such chemical reactions, there is always generating of heat. The most common fluid fluids like water and air are contaminated with impurities like $\mathrm{CO}_{2}$. Characterization of chemical reaction on heat transfer through the nano fluid has been addressed by Srikantha et al. [11]. Animasaun et al. [12] have elucidated the effects of thermophoresis, variable viscosity and thermal conductivity 
on free convective heat and mass transfer of non-darcian MHD dissipative Casson fluid flow with suction and nth order of chemical reaction. Numerical study of Williamson nanofluid flow in an asymmetric channel has been reported by Akbar et al. [13]. Their Problem has simplified with the reliable assumptions i.e. long wave length and small Reynolds number and numerical solutions have evaluated for stream function, velocity profile, temperature profile, nanoparticle phenomena and pressure rise. They have presented graphical results and discussed for various involved parameters. Also Ganga et al. [14] have adopted the MHD flow of Buongiorno model nanofluid over a vertical plate with internal heat generation/absorption. Unsteady Caisson nanofluid flow over a stretching sheet with thermal radiation, convective and slip boundary conditions has been discussed by Oyelakin et al. [15]. MHD free convection and mass transfer flow through a vertical oscillatory porous plate in a rotating porous medium with Hall, Ion-slip currents and heat source has been presented by Hossain et al. [16].

Motivated by the above studies, a purpose has been carried out to study the effects of chemical reaction on MHD unsteady heat and Mass transfer of nanofluid flow through a vertical plate. We have used a usual transformation to transform into non-dimensional form in our experiment and solved our problem numerically by using explicit finite different method. Also, our results are computed for various physical parameters and the obtained results are plotted after stability test. Further we observed, an increase in the magnetic parameter is to suppress the velocity but by increasing the heat generation parameter increased the temperature profiles.

\section{MATHEMATICAL ANALYSIS}

The unsteady two-dimensional laminar flow of nanofluids through an electrically conducting viscous incompressible semi-infinite vertical porous permeable plate $y=0$ has been considered where $x$-axis (plate axis) is taken along the plate in the vertically upward direction and the $y$-axis is taken normal to the plate which is displaced in the Fig.1. A uniform magnetic field of strength $B_{0}$ is applied transversely to the flow direction and induced magnetic field strength is neglected because of magnetic Reynolds number is taken very small. By using the relations $\nabla . \mathrm{B}=0$ or the magnetic field $\mathrm{B}=(0, \mathrm{~B} 0,0)$ has been consider everywhere in the fluid where $\mathrm{B} 0$ is constant. Also, it is considered that the temperature of the plate is assumed to be $\mathrm{Tw}$ and concentration at the plate is $\mathrm{Cw}$ at $\mathrm{t}=0$ but at time $\mathrm{t}>0$, the plate is accelerated exponentially with a velocity $\mathrm{u}=\mathrm{U} 0 \exp \left(\mathrm{b}^{\prime} \mathrm{t}\right)$ and the temperature and concentration level of the plate are increased exponentially to $T_{\infty}$ and $C_{\infty}$ with time $t$.

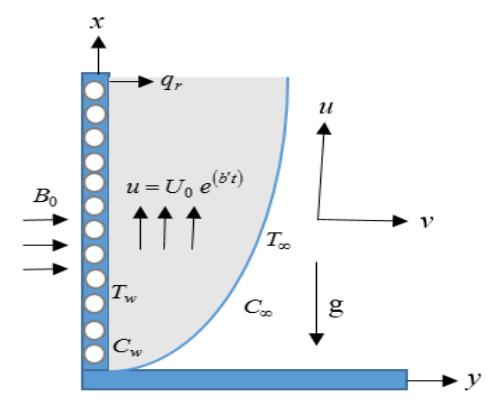

Under these assumptions the dimensional continuity, momentum, energy and concentration equations of the unsteady incompressible nanofluid boundary layer flow are as follows

$$
\frac{\partial u}{\partial x}+\frac{\partial v}{\partial y}=0
$$

$\frac{\partial u}{\partial t}+u \frac{\partial u}{\partial x}+v \frac{\partial u}{\partial y}=v \frac{\partial^{2} u}{\partial y^{2}}+g \beta_{T}\left(T-T_{\infty}\right)+g \beta_{C}\left(C-C_{\infty}\right)-\frac{v}{k^{*}} u-\frac{\sigma B_{0}^{2} u}{\rho}$

$\frac{\partial T}{\partial t}+u \frac{\partial T}{\partial x}+v \frac{\partial T}{\partial y}=\frac{k}{\rho C_{p}} \frac{\partial^{2} T}{\partial y^{2}}-\frac{1}{\rho C_{p}} \frac{\partial q_{r}}{\partial y}+\tau^{\prime}\left\{D_{B}\left(\frac{\partial C}{\partial y} \frac{\partial T}{\partial y}\right)+\frac{D_{T}}{T_{\infty}}\left(\frac{\partial T}{\partial y}\right)^{2}\right\}+\frac{Q}{\rho C_{p}}\left(T-T_{\infty}\right)$

$\frac{\partial C}{\partial t}+u \frac{\partial C}{\partial x}+v \frac{\partial C}{\partial y}=D_{m} \frac{\partial^{2} C}{\partial y^{2}}-K_{1}\left(C-C_{\infty}\right)+D_{B} \frac{\partial^{2} C}{\partial y^{2}}+\frac{D_{T}}{T_{\infty}} \frac{\partial^{2} T}{\partial y^{2}}+\frac{D_{m} K_{T}}{T_{m}} \frac{\partial^{2} T}{\partial y^{2}}$

The associate initial and boundary conditions for the present problem are,

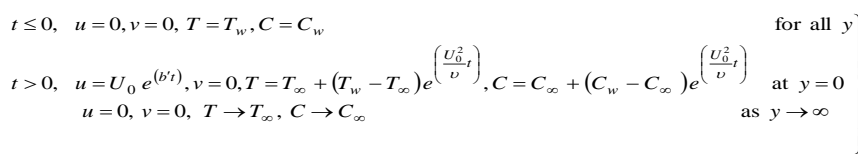

where, $u$ and $v$ are the velocity components in the $x$ and $y$-axis respectively, $v$ is the kinematic viscosity, $C_{p}$ is the specific heat at constant pressure, $k$ is the thermal conductivity, $\rho$ is the fluid density, $g$ is the gravitational acceleration, $k_{T}$ is the thermal diffusion ratio, $T$ is the temperature and $C$ is the concentration component of the fluid, $\beta_{T}$ is the thermal expansion coefficient, $\beta_{c}$ is the concentration expansion coefficient and $q_{r}$ is the radiated heat flux, $D_{B}$ is the Brownian diffusion coefficient, $\tau^{\prime}=(\rho c)_{p} /(\rho c)_{f}$ is the ratio of the heat capacity of the nanoparticle material and the heat capacity of the fluid, $D_{T}$ is the thermophoretic diffusion coefficient, $T_{m}$ is the mean fluid temperature and $k^{*}$ is the Darcy permeability.

Since the solution of the obtained governing equations under the initial and boundary conditions have been based on the finite difference method. It is to be required to make the equations dimensionless. For this purpose the following dimensionless variables are used.

$$
\begin{aligned}
& U=\frac{u}{U_{0}} ; V=\frac{v}{U_{0}} ; Y=\frac{y U_{0}}{v} ; X=\frac{x U_{0}}{v} ; \tau=\frac{t U_{0}^{2}}{v} ; \bar{T}=\frac{T-T_{\infty}}{T_{w}-T_{\infty}} ; \bar{C}=\frac{C-C_{\infty}}{C_{w}-c_{\infty}} \\
& S=\frac{Q v}{\rho C_{p} U_{0}^{2}} ; M=\frac{\sigma B_{0}^{2} v}{\rho U_{0}^{2}} ; S_{c}=\frac{v}{D_{m}} ; S_{r}=\frac{D_{m} K_{T}}{T_{m} v} \frac{\left(T_{w}-T_{\infty}\right)}{\left(C_{w}-C_{\infty}\right)} ; R_{a}=\frac{4 \sigma^{\prime} T_{\infty}^{3}}{k^{\prime} k} ; K_{p}=\frac{v^{2}}{k^{*} U_{0}^{2}} \\
& L_{e}=\frac{v}{D_{B}} ; \quad N_{t}=\frac{\tau^{\prime} D_{T}\left(T_{w}-T_{\infty}\right)}{T_{\infty} v} ; N_{b}=\frac{\tau^{\prime} D_{B}\left(C_{w}-C_{\infty}\right)}{v} ; \gamma=\frac{K_{1} v}{U_{0}^{2}} ; P_{r}=\frac{v \rho C_{p}}{k} \\
& G_{r}=\frac{v g \beta_{T}\left(T_{w}-T_{\infty}\right)}{U_{0}^{3}} ; G_{m}=\frac{v g \beta_{c}\left(C_{w}-C_{\infty}\right)}{U_{0}^{3}} ; b=\frac{b^{\prime} v}{U_{0}^{2}}
\end{aligned}
$$

Therefore, the dimensionless governing equations are

$\frac{\partial U}{\partial X}+\frac{\partial V}{\partial Y}=0$

Figure 1. Physical model and coordinate system 
$\frac{\partial U}{\partial \tau}+U \frac{\partial U}{\partial X}+V \frac{\partial U}{\partial Y}=\frac{\partial^{2} U}{\partial Y^{2}}+G_{r} \bar{T}+G_{m} \bar{C}-M U-K_{p} U$

$\frac{\partial \bar{T}}{\partial \tau}+U \frac{\partial \bar{T}}{\partial X}+V \frac{\partial \bar{T}}{\partial Y}=\frac{1}{P_{r}}\left(1+\frac{4}{3} R_{a}\right) \frac{\partial^{2} \bar{T}}{\partial Y^{2}}+S \bar{T}+N_{b} \frac{\partial \bar{C}}{\partial Y} \frac{\partial \bar{T}}{\partial Y}+N_{t}\left(\frac{\partial \bar{T}}{\partial Y}\right)^{2}$

$\frac{\partial \bar{C}}{\partial \tau}+U \frac{\partial \bar{C}}{\partial X}+V \frac{\partial \bar{C}}{\partial Y}=\frac{1}{S_{c}} \frac{\partial^{2} \bar{C}}{\partial Y^{2}}-\gamma \bar{C}+\frac{1}{L_{e}} \frac{\partial^{2} \bar{C}}{\partial Y^{2}}+\frac{N_{t}}{N_{b} L_{e}} \frac{\partial^{2} \bar{T}}{\partial Y^{2}}+S_{r} \frac{\partial^{2} \bar{T}}{\partial Y^{2}}$

Also, the associate boundary conditions after dimensionless of the present problem are,

$$
\left.\begin{array}{rl}
\tau>0, U & =\exp (b \tau), V=0, \bar{T}=\exp (\tau), \bar{C}=\exp (\tau) \text { at } Y=0 \\
U & =0, \bar{T} \rightarrow 0, \bar{C} \rightarrow 0 \quad \text { as } Y \rightarrow \infty
\end{array}\right\}
$$

where $M$ is magnetic parameter, $S_{C}$ is Schmidt number, $b$ is acceleration parameter, $\gamma$ is chemical reaction parameter, $P_{r}$ is Prandtl number, $G_{r}$ is Grashof number, $G_{m}$ is modified Grashof number, $R_{a}$ is radiation parameter, $N_{b}$ is Brownian Motion parameter, $N_{t}$ is thermophoresis parameter, $K_{P}$ is permeability of porous medium, $L_{e}$ is Lewis number, $S_{r}$ is Soret number and $S$ is heat source parameter.

The following quantities represents the skin friction coefficient, Nusselt number and Sherwood number at the plate,

$$
C_{f}=-\frac{1}{2 \sqrt{2}}\left(G_{r}\right)^{-\frac{3}{4}}\left(\frac{\partial U}{\partial Y}\right)_{Y=0} ; N_{u}=\frac{1}{\sqrt{2}}\left(G_{r}\right)^{-\frac{3}{4}}\left(\frac{\partial \bar{T}}{\partial Y}\right)_{Y=0} ; S_{h}=\frac{1}{\sqrt{2}}\left(G_{r}\right)^{-\frac{3}{4}}\left(\frac{\partial \bar{C}}{\partial Y}\right)_{Y=0}
$$

Continuity equation is satisfied by Stream function $\psi(X, Y)$ and which is associated with the velocity components as

$U=\frac{\partial \psi}{\partial Y}, V=-\frac{\partial \psi}{\partial X}$

\section{CALCULATION TECHNIQUE}

The finite difference equations for the equations (10)-(13) have been recounted by the nonlinear coupled partial differential equations (17) to (20) respectively as

$\frac{U_{i, j}-U_{i-1, j}}{\Delta X}+\frac{V_{i, j}-V_{i, j-1}}{\Delta Y}=0$

$\frac{U_{i, j}^{\prime}-U_{i, j}}{\Delta \tau}+U_{i, j}\left(\frac{U_{i, j}-U_{i-1, j}}{\Delta X}\right)+V_{i, j}\left(\frac{U_{i, j+1}-U_{i, j}}{\Delta \mathrm{Y}}\right)=\frac{U_{i, j+1}-2 U_{i, j}+U_{i, j-1}}{(\Delta \mathrm{Y})^{2}}+G_{r} \bar{T}_{i, j}$

$+G_{m} \bar{C}_{i, j}-M U_{i, j}-k_{p} U_{i, j}$

$\frac{\bar{T}_{i, j}^{\prime}-\bar{T}_{i, j}}{\Delta \tau}+U_{i, j} \frac{\bar{T}_{i, j}-\bar{T}_{i-1, j}}{\Delta X}+V_{i, j} \frac{\bar{T}_{i, j+1}-\bar{T}_{i, j}}{\Delta Y}=\frac{1}{P_{r}}\left(1+\frac{4}{3} R_{a}\right) \frac{\bar{T}_{i, j+1}-2 \bar{T}_{i, j}+\bar{T}_{i, j-1}}{(\Delta Y)^{2}}+S \bar{T}_{i, j}$

$+N_{b}\left(\frac{\bar{C}_{i, j+1}-\bar{C}_{i, j}}{\Delta Y}\right)\left(\frac{\bar{T}_{i, j+1}-\bar{T}_{i, j}}{\Delta Y}\right)+N_{t}\left(\frac{\bar{T}_{i, j+1}-\bar{T}_{i, j}}{\Delta Y}\right)^{2}$

$\frac{\bar{C}_{i, j}^{\prime}-C_{i, j}}{\Delta \tau}+U_{i, j} \frac{\bar{C}_{i, j}-\bar{C}_{i-1, j}}{\Delta X}+V_{i, j} \frac{\bar{C}_{i, j+1}-\bar{C}_{i, j}}{\Delta Y}=\left(\frac{1}{S_{c}}+\frac{1}{L_{e}}\right) \frac{\bar{C}_{i, j+1}-2 \bar{C}_{i, j}+\bar{C}_{i, j-1}}{(\Delta Y)^{2}}-\overline{C_{i, j}}$ $+\left(S_{r}+\frac{N_{t}}{N_{b} L_{e}}\right) \frac{\bar{T}_{i, j+1}-2 \bar{T}_{i, j}+\bar{T}_{i, j-1}}{(\Delta Y)^{2}}$

In this case, the associate boundary conditions are obtained as

$$
\left.\begin{array}{c}
\tau>0, U_{i, 0}^{n}=\exp (b n \Delta \tau), V_{i, 0}^{n}=0, \bar{T}_{i, 0}^{n}=\exp (n \Delta \tau), \bar{C}_{i, 0}^{n}=\exp (n \Delta \tau) \quad \text { at } \mathrm{Y}=0 \\
U_{i, L}^{n}=0, \bar{T}_{i, L}^{n} \rightarrow 0, \bar{C}_{i, L}^{n} \rightarrow 0 \text { where } L \rightarrow \infty
\end{array}\right\}
$$

Here $i$ and $j$ designate to the mesh points with $X$ and $Y$ coordinate respectively and the superscripts $n$ represent a value of time, $\tau=n \Delta \tau$ where, $n=0,1,2 \ldots$

\section{STABILITY AND CONVERGENCE ANALYSIS}

The stability conditions for the present problem are:

$\frac{2 \Delta \tau}{(\Delta Y)^{2}}+U \frac{\Delta \tau}{\Delta X}+|-V| \frac{\Delta \tau}{\Delta Y}+\frac{M \Delta \tau}{2}+\frac{K_{p} \Delta \tau}{2} \leq 1$

$\frac{1}{P_{r}}\left(1+\frac{4}{3} R_{a}\right) \frac{2 \Delta \tau}{(\Delta Y)^{2}}+U \frac{\Delta \tau}{\Delta X}+|-V| \frac{\Delta \tau}{\Delta Y}-\frac{S \Delta \tau}{2} \leq$

$\left(\frac{1}{S_{c}}+\frac{1}{L_{e}}\right) \frac{2 \Delta \tau}{(\Delta Y)^{2}}+U \frac{\Delta \tau}{\Delta X}+|-V| \frac{\Delta \tau}{\Delta Y}+\frac{\gamma \Delta \tau}{2} \leq 1$

Using initial condition, $U=V=0$ at $\tau=0$ in the equations (22) to (24), then the convergence criteria of the present problem are obtained as $P_{r} \geq 0.038$ and $S_{c} \geq 0.028$ respectively.

Therefore, the convergence criteria of the present problem are $P_{r} \geq 0.038$ and $S_{c} \geq 0.028$.

\section{RESULTS AND DISCUSSION}

In order to investigate the results of the present problem, a finite difference solution is used by explicit finite difference method. The numerical values of non-dimensional velocity, temperature, concentration, skin friction coefficient, Nusselt number, Sherwood number, streamlines and isotherms for vertical plate within the boundary conditions are obtained by FORTRAN program in the variations of different values of different parameters such as magnetic parameter $(M)$, permeability of porous medium $\left(K_{p}\right)$, Schmidt number $\left(S_{c}\right)$, Prandtl number $\left(P_{r}\right)$, chemical reaction parameter $(\gamma)$, Grashof number $\left(G_{r}\right)$, modified Grashof number $\left(G_{m}\right)$, heat source parameter $(\mathrm{S})$, Soret number $\left(S_{r}\right)$, thermophoresis parameter $\left(N_{t}\right)$, Brownian motion parameter $\left(N_{b}\right)$ and radiation parameter $\left(R_{a}\right)$ which are shown in Figs. 2 to 23 . In order to obtain the accuracy of the numerical results, the following values of default parameter are chosen as: $M=1.0, P_{r}=1.0, S_{c}=0.22$, $R_{a}=0.50, S=0.1, N_{b}=0.5, N_{t}=0.8, L_{e}=2.5, S_{r}=2, G_{m}=5, G_{r}=10$, $K_{p}=1$, and $\gamma=0.50$ with time $\tau=1$. These values are treated as same throughout the study in respective Figs and Table 1.

The outcome of different values of permeability of porous medium, chemical reaction parameter, Grashof number, magnetic parameter, Soret number, radiation parameter, Brownian motion parameter, heat source parameter and modified Grashof number on velocity profiles are displayed in the Figs. 2-10 respectively. It is examined that velocity profiles are increasing respectively as Grashof number and modified Grashof number which are shown in Fig. 4 and Fig. 10. The thermal Grashof number which signifies the relative effect of the thermal buoyancy force in the boundary layer. Due to this augmentation of thermal buoyancy force which executing on the fluid particles for gravitational force that increase of the thickness of momentum boundary layer. This is the fact that 
velocity is increasing due to increase of Grashof number. Also a similar effect is visible in the vicinity of modified Grashof number. Also, velocity profiles are decreasing 34.6\%, 23.6\%, $20.8 \%$ and $12.2 \%$ as permeability of porous medium changes as 1.00 to $2.00,2.00$ to $3.00,3.00$ to 4.00 and 4.00 to 5.00 respectively which occurs in the Fig. 2. Physically, the permeability of porous medium $\mathrm{Kp}$ which increases the resistive force. For this resistive force, velocity profiles are decreasing with the increasing of permeability of porous medium. In the Fig. 5, the impact of different values of magnetic parameter $\mathrm{M}$ on velocity profiles are exhibited. It is watched that velocity decreased by $59.49 \%, 32.50 \%, 20.25 \%$, and $13.95 \%$ for $M=0.50$ to $M=1.50, M=1.50$ to $M=2.50$, $M=2.50$ to $M=3.50$ and $M=3.50$ to $M=4.50$ respectively. The decrease in the tangential velocity as the increase of magnetic field which introduce a resistive force called Lorentz force. This force is one kind of resistive force which slows down the fluid velocity. Also, the velocity profiles are exhibited in Figs. $(6,7,9)$ respectively for different values of Soret number, radiation parameter and heat source parameter. It is demonstrated that there is an increment in the velocity for the increased of all these parameters. This may happen due to an increase of radiation parameter which enhance the thermal boundary layer. Further it is noted that for assisting flow, fluid velocity decrease for increasing values of chemical reaction and Brownian motion parameter which are shown in Fig. 3 and Fig. 8 respectively.

The effects of Brownian motion parameter, radiation parameter, Prandtl number, thermophoresis parameter and heat source parameter on temperature profiles are displaced in Figs. 11-15. It is worth mentioned that thermophoresis parameter and Brownian motion parameter are coupled with the temperature field and we observed that the temperature of the fluid decreased with the increase of Brownian motion parameter but increased considerable with the increase of thermophoresis parameter which are shown in Fig. 11 and Fig. 14. This is due to fact that, the unrestrained motion of nanoparticles gets increased with an increase in thermophoresis parameter which enhancement of fluid temperature. Here it is adopted that temperature profiles are increased by $34.49 \%, 25.50 \%, 24.25 \%$, and $16.95 \%$ for $R_{a}=0.50$ to $R_{a}=1.50, R_{a}=1.50$ to $R_{a}=2.50, R_{a}=2.50$ to $R_{a}=3.50$ and $R_{a}=3.50$ to $R_{a}=4.50$ respectively which are shown in Fig. 12. Physically, radiation parameter Ra provides more heat into the fluid, which leads to increases the thermal boundary layer thickness by increasing the values of radiation parameter. Also, temperature profiles decreased by Prandtl number but increased by heat source parameter which are shown in Fig. 12 and Fig. 15, respectively.

The impact of Lewis number, thermophoresis parameter, Soret number and chemical reaction on concentration profiles are shown in Figs. 16-19. We capitalized from Fig. 17 and Fig. 18 that concentration profiles increase to the increasing of thermophoresis parameter and Soret number where thermophoresis parameter leads the concentration profiles but Lewis number and chemical reaction decreased the concentration profiles which are shown in Fig. 16 and Fig. 19 respectively. Physically, it is mentioned that thermophoresis parameter enhancement the nanoparticle. The influence of magnetic parameter $\mathrm{M}$ on streamlines and isotherms are showed in Figs. 20-21. It is capitalized that momentum boundary layer thickness increases due to the increase of magnetic parameter from $M=0.50$ to $M=1.50$ but thermal boundary layer thickness decreases by the increasing of magnetic $n$ parameter from $M=0.50$ to $M=1.50$. Also, the outcome of radiation parameter $\mathrm{Ra}$ on streamlines and isotherms are scrutinized in Figs. 25 to 26. Here, we carried out that both momentum and thermal boundary layer thickness are increasing for increasing of radiation parameter from $R_{a}=0.50$ to $R_{a}=1.50$.

Table 1 represents the numerical values of different parameters such as Soret number $(\mathrm{Sr})$, magnetic parameter $(\mathrm{M})$ radiation parameter $(\mathrm{Ra})$, chemical reaction parameter $(\gamma)$, Lewis number $\left(L_{e}\right)$, thermophoresis parameter $\left(N_{t}\right)$, Brownian motion parameter $\left(N_{b}\right)$ and Prandtl number $\left(P_{r}\right)$ on skin friction coefficient $\left(C_{f}\right)$, Nusselt number $\left(N_{u}\right)$ and Sherwood number $\left(S_{h}\right)$ for nanofluid flow. It is observed that the skin friction coefficient decreases with magnetic parameter $(M)$, chemical reaction parameter $(\gamma)$, Lewis number $\left(L_{e}\right)$, Brownian motion parameter $\left(N_{b}\right)$ and Prandtl number $\left(P_{r}\right)$ but increases qualitatively with an increase in Soret number $\left(S_{r}\right)$, radiation parameter $\left(R_{a}\right)$ and thermophoresis parameter $\left(N_{t}\right)$. On the other hand, the Nusselt number is an increasing functions of chemical reaction parameter $(\gamma)$, Lewis number $\left(L_{e}\right)$, Brownian motion parameter $\left(N_{b}\right)$ and Prandtl number $\left(P_{r}\right)$ whereas this tendency is quite opposite for thermophoresis parameter $\left(N_{t}\right)$, radiation parameter $\left(R_{a}\right)$ and Soret number $\left(S_{r}\right)$. Also, it is noticed that the Sherwood number increases with the increasing of radiation parameter $\left(R_{a}\right)$, chemical reaction parameter $(\gamma)$ and Lewis number $\left(L_{e}\right)$ but decreases qualitatively with the increasing of in Soret number $\left(S_{r}\right)$, thermophoresis parameter $\left(N_{t}\right)$, Brownian motion parameter $\left(N_{b}\right)$ and Prandtl number $\left(P_{r}\right)$.

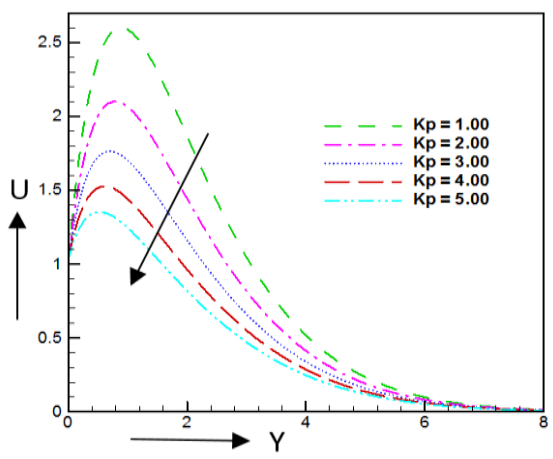

Figure 2. Velocity profiles for different values of $K_{p}$ against $Y$ when $M=1.0, P_{r}=1.0, S_{c}=0.22, R_{a}=0.50, S_{r}=2.0, S=0.10$, $\gamma=0.50, N_{t}=0.8, N_{b}=0.5$ and $L_{e}=2.5$

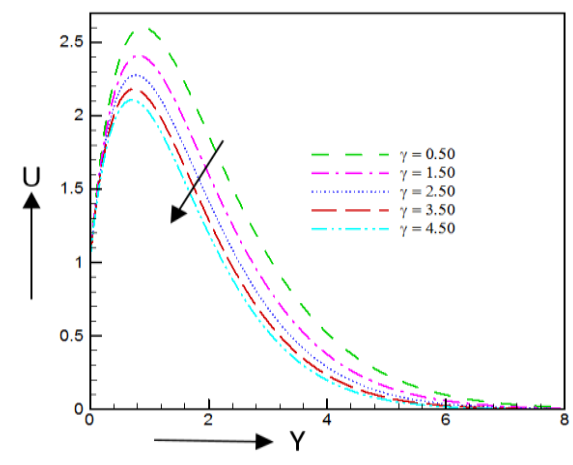

Figure 3. Velocity profiles for different values of against $\mathrm{Y}$ when $\mathrm{M}=1.0, \mathrm{Pr}=1.0, \mathrm{Sc}=0.22, \mathrm{Ra}=0.50, \mathrm{Sr}=2.0, \mathrm{~S}=0.10$, $\mathrm{Kp}=1.0, \mathrm{Nt}=0.8, \mathrm{Nb}=0.5$ and $\mathrm{Le}=2.5$ 


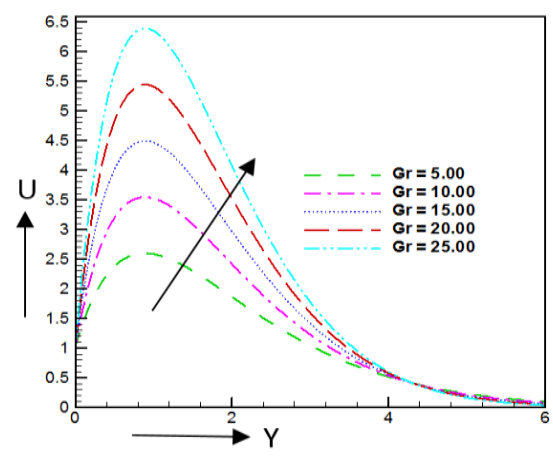

Figure 4. Velocity profiles for different values of $\mathrm{Gr}$ against $\mathrm{Y}$ when $\mathrm{M}=1.0, \mathrm{Pr}=1.0, \mathrm{Sc}=0.22, \mathrm{Ra}=0.50, \mathrm{Sr}=2.0, \mathrm{~S}=0.10$, $=0.50, \mathrm{Nt}=0.8, \mathrm{Nb}=0.5$ and $\mathrm{Le}=2.5$

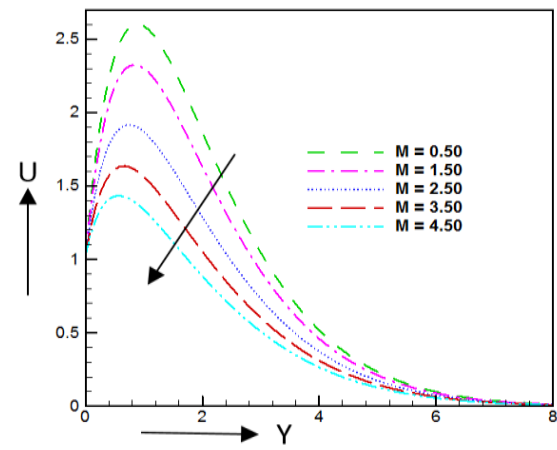

Figure 5. Velocity profiles for different values of $\mathrm{M}$ against $\mathrm{Y}$ when $\mathrm{Kp}=1.0, \mathrm{Pr}=1.0, \mathrm{Sc}=0.22, \mathrm{Ra}=0.50, \mathrm{Sr}=2.0, \mathrm{~S}=0.10$, $=0.50, \mathrm{Nt}=0.8, \mathrm{Nb}=0.5$ and $\mathrm{Le}=2.5$

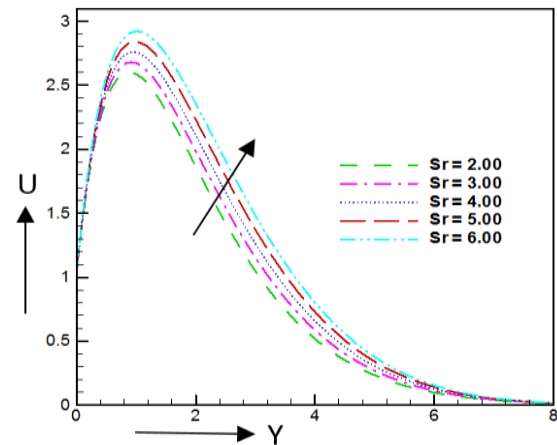

Figure 6. Velocity profiles for different values of $\mathrm{Sr}$ against $\mathrm{Y}$ when $\mathrm{M}=1.0, \mathrm{Pr}=1.0, \mathrm{Sc}=0.22, \mathrm{Ra}=0.50, \mathrm{Kp}=1.0, \mathrm{~S}=0.10$, $=0.50, \mathrm{Nt}=0.8, \mathrm{Nb}=0.5$ and $\mathrm{Le}=2.5$

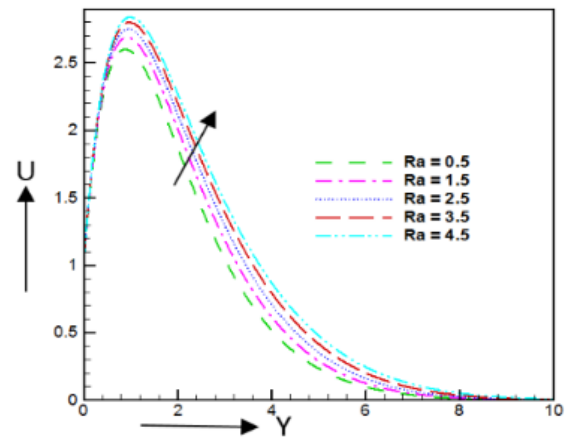

Figure 7. Velocity profiles for different values of Ra against $\mathrm{Y}$ when $\mathrm{M}=1.0, \mathrm{Pr}=1.0, \mathrm{Sc}=0.22, \mathrm{Kp}=1.0, \mathrm{Sr}=2.0, \mathrm{~S}=0.10$, $=0.50, \mathrm{Nt}=0.8, \mathrm{Nb}=0.5$ and $\mathrm{Le}=2.5$

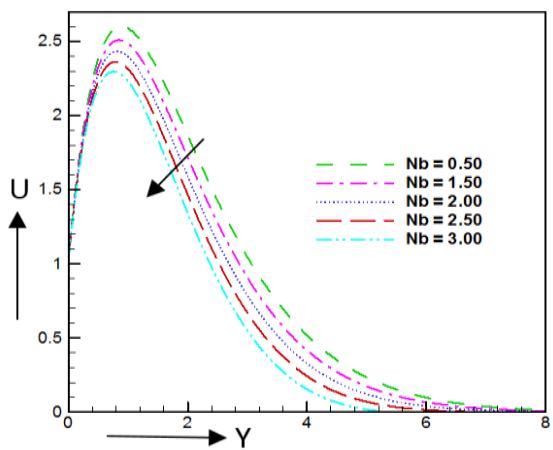

Figure 8. Velocity profiles for different values of $\mathrm{Nb}$ against $\mathrm{Y}$ when $\mathrm{M}=1.0, \mathrm{Pr}=1.0, \mathrm{Sc}=0.22, \mathrm{Ra}=0.50, \mathrm{Sr}=2.0, \mathrm{~S}=0.10$, $=0.50, \mathrm{Nt}=0.8, \mathrm{Kp}=1.0$ and $\mathrm{Le}=2.5$

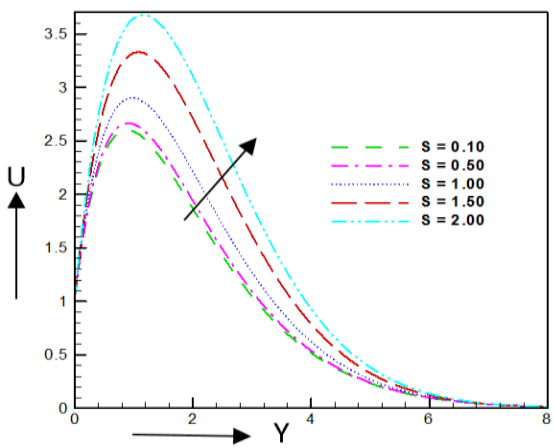

Figure 9. Velocity profiles for different values of $S$ against $Y$ when $M=1.0, P_{r}=1.0, S_{c}=0.22, R_{a}=0.50, S_{r}=2.0, K_{p}=1.0$, $\gamma=0.50, N_{t}=0.8, N_{b}=0.5$ and $L_{e}=2.5$

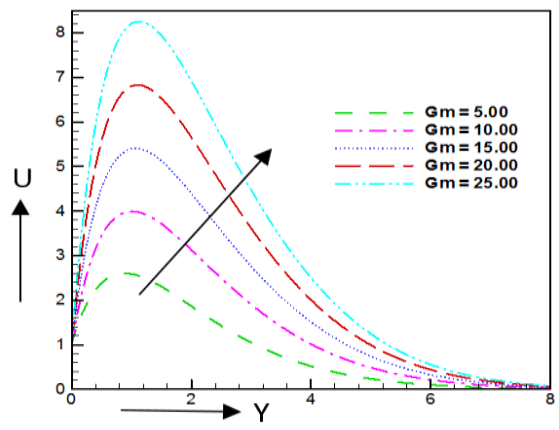

Figure 10. Velocity profiles for different values of $\mathrm{Gm}$ against $\mathrm{Y}$ when $\mathrm{M}=1.0, \mathrm{Pr}=1.0, \mathrm{Sc}=0.22, \mathrm{Ra}=0.50, \mathrm{Sr}=2.0$, $\mathrm{S}=0.10, \quad=0.50, \mathrm{Nt}=0.8, \mathrm{Nb}=0.5$ and $\mathrm{Le}=2.5$

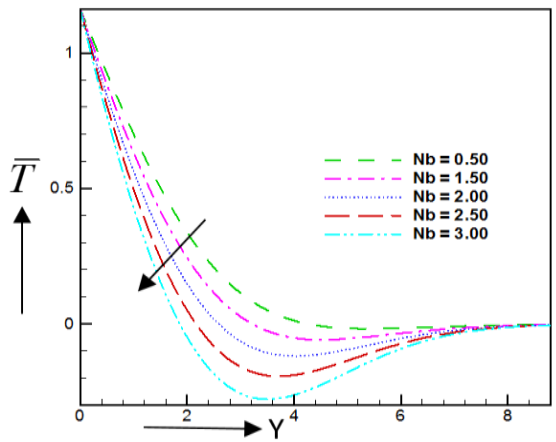

Figure 11. Temperature profiles for different values of $N_{b}$ against $Y$ when $M=1.0, P_{r}=1.0, S_{c}=0.22, R_{a}=0.50, S_{r}=2.0$, $S=0.10, \gamma=0.50, N_{t}=0.8, K_{p}=1.0$ and $L_{e}=2.5$ 


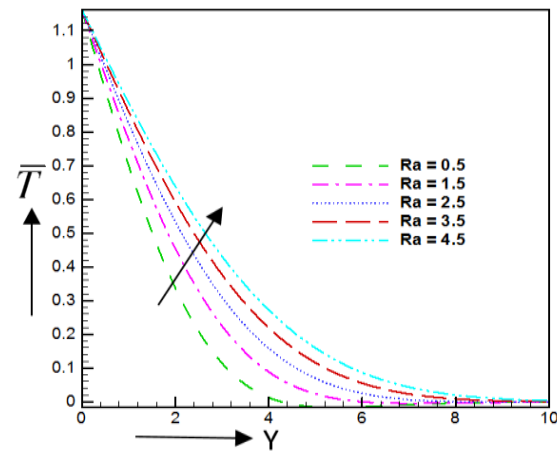

Figure 12. Temperature profiles for different values of $R_{a}$ against $Y$ when $M=1.0, P_{r}=1.0, S_{c}=0.22, K_{p}=1.0, S_{r}=2.0$, $S=0.10, \gamma=0.50, N_{t}=0.8, N_{b}=0.5$ and $L_{e}=2.5$

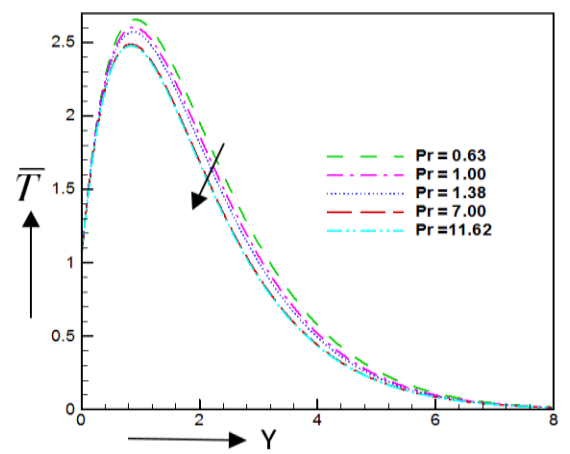

Figure 13. Temperature profiles for different values of $P_{r}$ against $Y$ when $M=1.0, K_{p}=1.0, S_{c}=0.22, R_{a}=0.50, S_{r}=2.0$, $S=0.10, \gamma=0.50, N_{t}=0.8, N_{b}=0.5$ and $L_{e}=2.5$

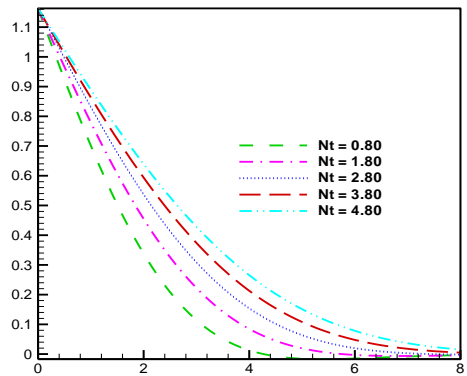

Figure 14. Temperature profiles for different values of $\mathrm{Nt}$ against $\mathrm{Y}$ when $\mathrm{M}=1.0, \mathrm{Pr}=1.0, \mathrm{Sc}=0.22, \mathrm{Ra}=0.50, \mathrm{Sr}=2.0$, $\mathrm{S}=0.10, \quad=0.50, \mathrm{Kp}=1.0, \mathrm{Nb}=0.5$ and $\mathrm{Le}=2.5$

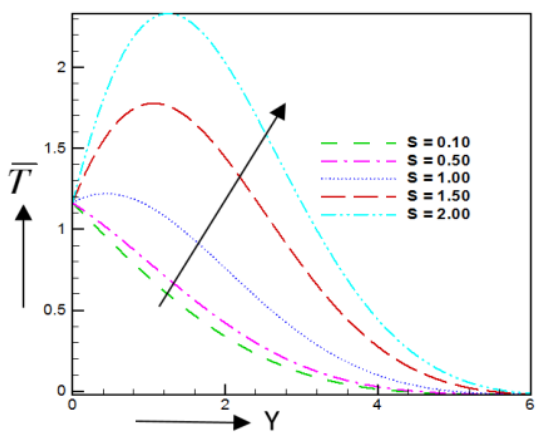

Figure 15. Temperature profiles for different values of $S$ against $Y$ when $M=1.0, P_{r}=1.0, S_{c}=0.22, R_{a}=0.50, S_{r}=2.0, K_{p}$ $=1.0, \gamma=0.50, N_{t}=0.8, N_{b}=0.5$ and $L_{e}=2.5$

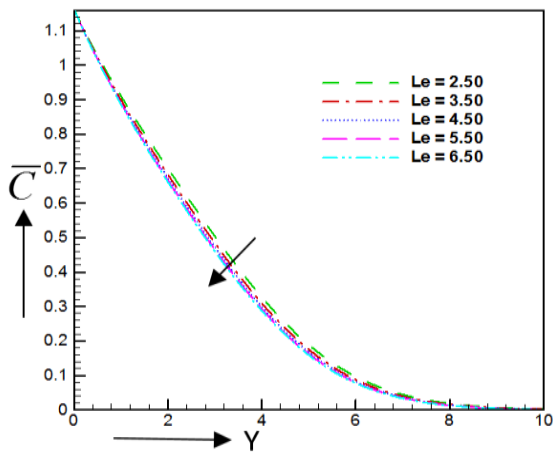

Figure 16. Concentration profiles for different values of $L_{e}$ against $Y$ when $M=1.0, P_{r}=1.0, S_{c}=0.22, R_{a}=0.50, S_{r}=2.0$, $S=0.10, \gamma=0.50, N_{t}=0.8, N_{b}=0.5$ and $K_{p}=1.0$

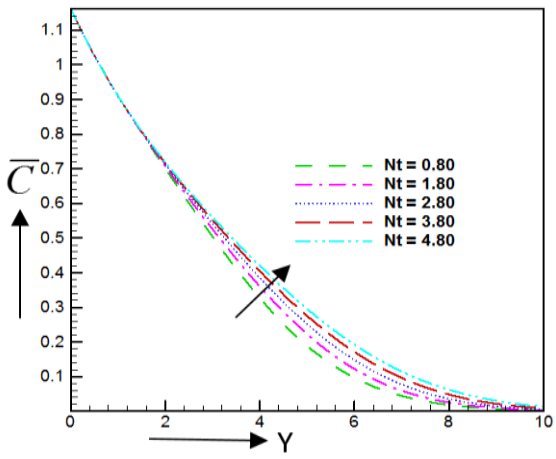

Figure 17. Concentration profiles for different values of $\mathrm{Nt}$ against $\mathrm{Y}$ when $\mathrm{M}=1.0, \mathrm{Pr}=1.0, \mathrm{Sc}=0.22, \mathrm{Ra}=0.50, \mathrm{Sr}=2.0$, $\mathrm{S}=0.10, \quad=0.50, \mathrm{Kp}=1.0, \mathrm{Nb}=0.5$ and $\mathrm{Le}=2.5$

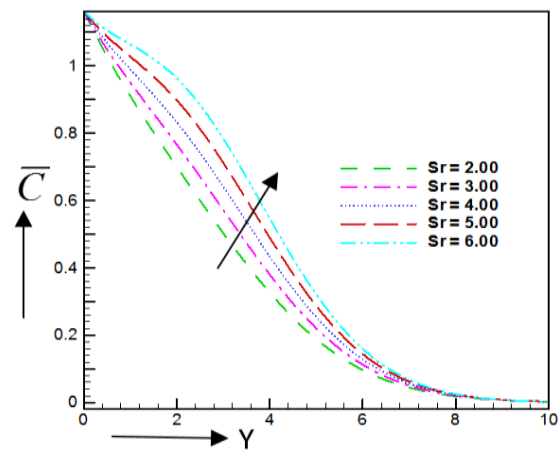

Figure 18. Concentration profiles for different values of $S_{r}$ against $Y$ when $M=1.0, P_{r}=1.0, S_{c}=0.22, R_{a}=0.50, K_{p}=1.0$, $S=0.10, \gamma=0.50$

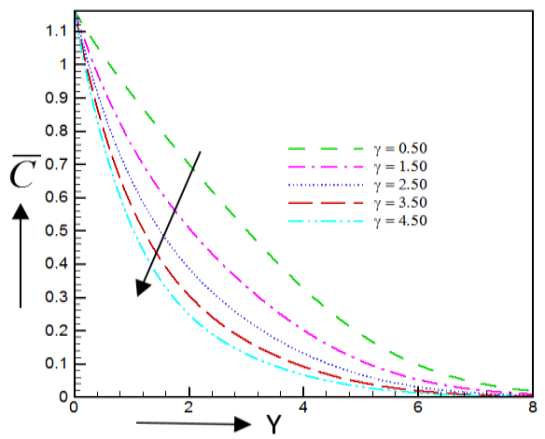

Figure 19. Concentration profiles for different values of $\gamma$ against $Y$ when $M=1.0, P_{r}=1.0, S_{c}=0.22, R_{a}=0.50, S_{r}=2.0$, $S=0.10, K_{p}=1.0$ 


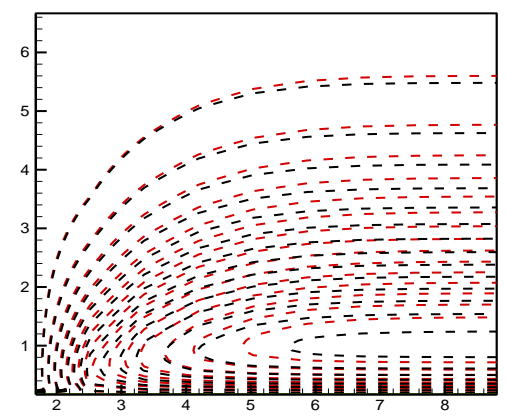

Figure 20. Streamlines for $M=0.50$ (red dashed line) and $M=1.00$ (black dashed line)

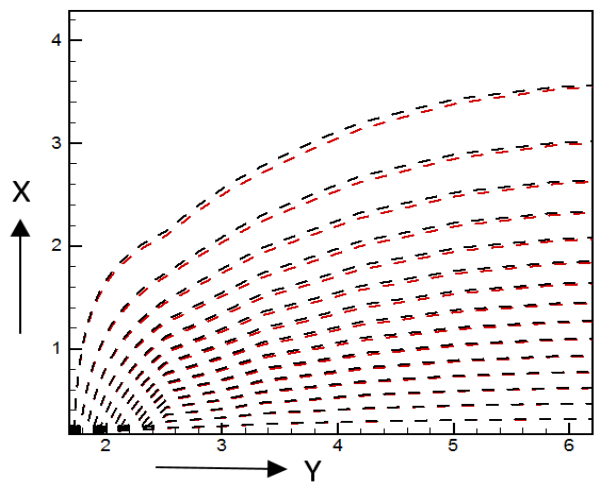

Figure 21. Isotherms for $M=0.50$ (red dashed line) and $M=1.00$ (black dashed line)

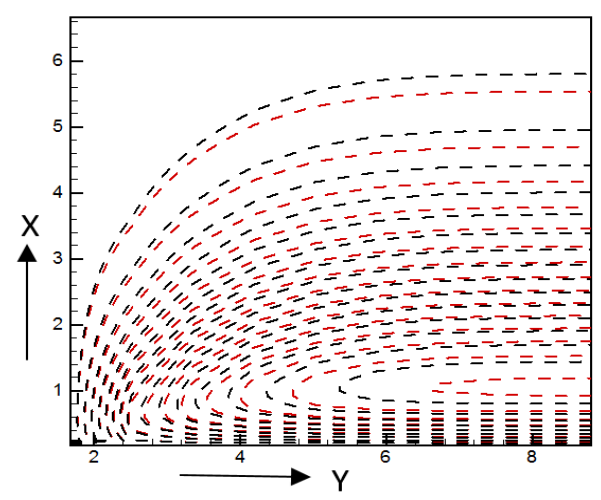

Figure 22. Streamlines for $R_{a}=0.50$ (red dashed line) and $R_{a}$ $=1.50$ (black dashed line)

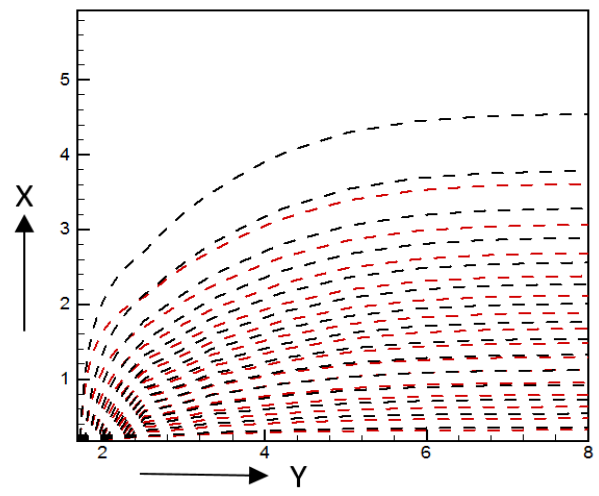

Figure 23. Isotherms for $\mathrm{Ra}=0.50$ (red dashed line) and $\mathrm{Ra}$ $=1.50$ (black dashed line)

Table 1. Variation of different parameters on local skin friction, Nusselt number and Sherwood number for nanofluid.

\begin{tabular}{ccccccccccc}
\hline$S_{r}$ & $M$ & $R_{a}$ & $\gamma$ & $L e$ & $N_{t}$ & $N_{b}$ & $P r$ & $C_{f}$ & $N_{u}$ & $S_{h}$ \\
\hline 2.00 & 1.00 & 0.50 & 0.50 & 2.50 & 0.80 & 0.50 & 0.63 & -0.03412 & 0.70393 & 0.24734 \\
3.50 & 1.00 & 0.50 & 0.50 & 2.50 & 0.80 & 0.50 & 0.63 & -0.02254 & 0.70076 & 0.16988 \\
4.00 & 1.00 & 0.50 & 0.50 & 2.50 & 0.80 & 0.50 & 0.63 & -0.01100 & 0.69758 & 0.09363 \\
2.00 & 1.00 & 0.50 & 0.50 & 2.50 & 0.80 & 0.50 & 0.63 & -0.00259 & 0.67977 & 0.23986 \\
2.00 & 1.50 & 0.50 & 0.50 & 2.50 & 0.80 & 0.50 & 0.63 & -0.02282 & 0.67977 & 0.23986 \\
2.00 & 2.00 & 0.50 & 0.50 & 2.50 & 0.80 & 0.50 & 0.63 & -0.06238 & 0.67977 & 0.23986 \\
2.00 & 1.00 & 0.50 & 0.50 & 2.50 & 0.80 & 0.50 & 0.63 & 0.71930 & 0.29348 & 0.13451 \\
2.00 & 1.00 & 1.50 & 0.50 & 2.50 & 0.80 & 0.50 & 0.63 & 0.73949 & 0.24418 & 0.15331 \\
2.00 & 1.00 & 2.50 & 0.50 & 2.50 & 0.80 & 0.50 & 0.63 & 0.75367 & 0.21305 & 0.16463 \\
2.00 & 1.00 & 0.50 & 0.50 & 2.50 & 0.80 & 0.50 & 0.63 & 0.71032 & 0.29684 & 0.13514 \\
2.00 & 1.00 & 0.50 & 1.50 & 2.50 & 0.80 & 0.50 & 0.63 & 0.68433 & 0.30244 & 0.21417 \\
2.00 & 1.00 & 0.50 & 2.50 & 2.50 & 0.80 & 0.50 & 0.63 & 0.66181 & 0.30701 & 0.28288 \\
2.00 & 1.00 & 0.50 & 0.50 & 2.50 & 0.80 & 0.50 & 0.63 & 0.71566 & 0.29484 & 0.13476 \\
2.00 & 1.00 & 0.50 & 0.50 & 3.50 & 0.80 & 0.50 & 0.63 & 0.71025 & 0.29578 & 0.14217 \\
2.00 & 1.00 & 0.50 & 0.50 & 4.50 & 0.80 & 0.50 & 0.63 & 0.70711 & 0.29633 & 0.14651 \\
2.00 & 1.00 & 0.50 & 0.50 & 2.50 & 0.80 & 0.50 & 0.63 & -0.16786 & 0.81388 & 0.28169 \\
2.00 & 1.00 & 0.50 & 0.50 & 2.50 & 1.80 & 0.50 & 0.63 & -0.15187 & 0.67535 & 0.28139 \\
2.00 & 1.00 & 0.50 & 0.50 & 2.50 & 2.80 & 0.50 & 0.63 & -0.13163 & 0.52922 & 0.27520 \\
2.00 & 1.00 & 0.50 & 0.50 & 2.50 & 0.80 & 0.50 & 0.63 & 0.72880 & 0.28995 & 0.13387 \\
2.00 & 1.00 & 0.50 & 0.50 & 2.50 & 0.80 & 1.00 & 0.63 & 0.71120 & 0.32676 & 0.13190 \\
2.00 & 1.00 & 0.50 & 0.50 & 2.50 & 0.80 & 1.50 & 0.63 & 0.69765 & 0.36239 & 0.12502 \\
2.00 & 1.00 & 0.50 & 0.50 & 2.50 & 0.80 & 0.50 & 0.63 & -0.07189 & 0.65809 & 0.29133 \\
2.00 & 1.00 & 0.50 & 0.50 & 2.50 & 0.80 & 0.50 & 1.00 & -0.07828 & 0.73892 & 0.25823 \\
2.00 & 1.00 & 0.50 & 0.50 & 2.50 & 0.80 & 0.50 & 1.38 & -0.08190 & 0.78850 & 0.23750 \\
\hline & & & & & & & & & &
\end{tabular}




\section{CONCLUSION}

From the present numerical investigation, following conclusions have been drawn:

(1). The skin friction coefficient decreases with magnetic parameter, chemical reaction parameter, Lewis number, Brownian motion parameter and Prandtl number.

(2). It is noticed that Grashof number and modified Grashof number increases the velocity profiles.

(3). Nusselt number is an increasing functions of chemical reaction parameter, Lewis number, Brownian motion parameter and Prandtl number.

(4). It is observed that permeability of porous medium and magnetic parameter decreases the velocity.

(5). Radiation parameter increase the temperature profiles.

(6). In view of that Sherwood number decreases qualitatively with the increasing of in Soret number, thermophoresis parameter, Brownian motion parameter and Prandtl number.

\section{REFERENCES}

[1] Choi S. (1995). Enhancing thermal conductivity of fluids with nanoparticles. ASME-Publ. Fed (231): 99-106. https://www.researchgate.net/profile/Jeffrey_Eastman/p ublication/236353373_Enhancing_thermal_conductivity of fluids with nanoparticles/links/0f3175336e78aa9c 4c000000/Enhancing-thermal-conductivity-of-fluidswith-nanoparticles.pdf

[2] Uddin MJ, Kalbani KSA, Rahman MM, Alam MS, Salti NA, Eltayeb IA. (2016). Fundamentals of nanofluids: evolution, applications and new theory. Official Journal of Biomathematical Society of India 2(1): 1-32.

[3] Mahanthesh B, Gireesha BJ, Gorla RSR. (2016). Unsteady three-dimensional MHD flow of a nano Eyring-Powell fluid past a convectively heated stretching sheet in the presence of thermal radiation, viscous dissipation and Joule heating. Journal of the Associatin of Arab Universities for Basic and Applied Sciences 7584. http://doi.org/10.1016/j.jaubas.2016.05.004

[4] Khan NA, Sultan F, Rubbab Q. (2015). Optimal solution of nonlinear heat and mass transfer in a two-layer flow with nano-Eyring-Powell fluid. Results in Physics 5: 199-205. http://doi.org/10.1016/j.rinp.2015.08.006

[5] Hossain MD, Samad MA, Alam MM. (2015). MHD free convection and mass transfer flow through a vertical oscillatory porous plate with hall, ion-slip currents and heat source in a rotating system. Procedia Engineering 105: 56-63. http://doi.org/10.1016/j.proeng.2015.05.006

[6] Das S, Guchhait SK, Jana RN, Makinde OD. (2016). Hall effects on an unsteady magneto-convection and radiative heat transfer past a porous plate. Alexandria Engineering Journal 55: 1321-1331.

[7] Seth GS, Sarkar S, Hussain SM. (2014). Effects of hall current, radiation and rotation on natural convection heat and mass transfer flow past a moving vertical plate. Ain Shams Engineering Journal 5: 489-503. http://doi.org/10.1016/j.asej.2013.09.014

[8] Biswas R, Mondal M, Sarkar DR, Ahmmed SF. (2017). Effects of radiation and chemical reaction on MHD unsteady heat and mass transfer of Casson fluid flow past a vertical plate. Journal of Advances in Mathematics and Computer Science, 23(2), 1-16. http://doi.org/10.9734/JAMCS/2017/34292

[9] Haque Z, Alam MM. (2011). Micropolar fluid behaviours on unsteady MHD heat and Mass transfer flow with constant heat and mass fluxes, joule heating and viscous dissipation. AMSE Journal 80(2).

[10] Haque M, Alam MM. (2009). Transient heat and mass transfer by mixed convection flow from a vertical porous plate with induced magnetic field. constant heat and mass fluxes. AMSE Journal 78(4).

[11] Srikantha GVPN, Srinivasa DG, Babub BS. (2015). Characterization of chemical reaction on heat transfer through the nano fluid. Procedia Materials Science 10: 10-18. http://doi.org/10.1016/j.mspro.2015.06.018

[12] Animasaun IL. (2015). Effects of thermophoresis, variable viscosity and thermal conductivity on free convective heat and mass transfer of non-Darcian MHD dissipative Casson fluid flow with suction and nth order of chemical reaction. Journal of the Nigerian Mathematical Society 34: 11-31. http://doi.org/10.1016/j.jnnms.2014.10.008

[13] Akbar NS, Nadeem S, Lee C, Khan ZH, Haque RU. (2013). Numerical study of Williamson nano fluid flow in an asymmetric channel. Results in Physics 3: 161-166. http://doi.org/10.1016/j.rinp.2013.08.005

[14] Ganga B, Ansari SMY, Ganeshc NV, Hakeem AKA. (2016). MHD flow of Boungiorno model nanofluid over a vertical plate with internal heat generation/absorption. Propulsin and Power Research 5(3): 211-222. http://doi.org/10.1016/j.jppr.2016.07.003

[15] Oyelakin IS., Mondal S, Sibanda P. (2016). Unsteady Casson nanofluid flow over a stretching sheet with thermal radiation. convective and slip boundary conditions. Alexandria Engineering Journal 55: 10251035. http://doi.org/10.1016/j.aej.2016.03.003

[16] Hossain MD, Samad MA, Alam MM. (2016). MHD Free Convection and Mass Transfer Flow through a Vertical Oscillatory Porous Plate in a Rotating Porous Medium with Hall, Ion-Slip Currents and Heat Source. AMSE Journals -2016-Series: Modelling B 85(1): 28-42. http://doi.org/10.1016/j.proeng.2015.05.006 